\title{
Kapitza-Dirac Blockade: A Universal Tool for the Deterministic Preparation of Non-Gaussian Oscillator States
}

\author{
Wayne Cheng-Wei Huang $\odot{ }^{1,{ }^{*}}$ Herman Batelaan $\odot,{ }^{2}$ and Markus Arndt $\oplus^{3}$ \\ ${ }^{1}$ Department of Physics and Astronomy, Northwestern University, Evanston, Illinois 60208, USA \\ ${ }^{2}$ Department of Physics and Astronomy, University of Nebraska-Lincoln, Lincoln, Nebraska 68588, USA \\ ${ }^{3}$ Faculty of Physics, University of Vienna, Boltzmanngasse 5, A-1090 Vienna, Austria
}

(Received 12 September 2020; revised 20 December 2020; accepted 24 May 2021; published 23 June 2021; corrected 29 June 2021)

\begin{abstract}
Harmonic oscillators count among the most fundamental quantum systems with important applications in molecular physics, nanoparticle trapping, and quantum information processing. Their equidistant energy level spacing is often a desired feature, but at the same time a challenge if the goal is to deterministically populate specific eigenstates. Here, we show how interference in the transition amplitudes in a bichromatic laser field can suppress the sequential climbing of harmonic oscillator states (Kapitza-Dirac blockade) and achieve selective excitation of energy eigenstates, cat states, and other non-Gaussian states. This technique can transform the harmonic oscillator into a coherent two-level system or be used to build a largemomentum-transfer beam splitter for matter waves. To illustrate the universality of the concept, we discuss feasible experiments that cover many orders of magnitude in mass, from single electrons over large molecules to dielectric nanoparticles.
\end{abstract}

DOI: 10.1103/PhysRevLett.126.253601

The harmonic oscillator is a paradigmatic textbook example of fundamental quantum physics and it has remained at the heart of modern research. Quantum harmonic oscillators have been realized with single electrons [1,2], single ions [3], ultracold quantum gases [4], and dielectric nanoparticles $[5,6]$. For all these systems, cooling to the oscillator ground state has been successfully demonstrated. Our present proposal is motivated by the challenge to prepare highly nonclassical states, mesoscopic cat states, and large-momentum-transfer beam splitters, independent of detailed oscillator properties.

Throughout the last two decades, macroscopic quantum superposition was realized in widely different systems [7]. Neutrons were delocalized over $10 \mathrm{~cm}$ [8]. Atoms were put in superpositions on the half-meter scale [9] or in momentum states separated by more than $1000 \hbar k$ [10], and molecules in excess of 25000 Da were delocalized over a hundred times their size [11]. Lately, it has been proposed to prepare dielectric nanoparticles in distinct position states $[7,12]$ to test the nature of quantum collapse [13], quantum decoherence [14-16], or even the quantum nature of gravity $[17,18]$.

Here we propose to exploit the Kapitza-Dirac blockade as a universal tool for preparing cat states or non-Gaussian

Published by the American Physical Society under the terms of the Creative Commons Attribution 4.0 International license. Further distribution of this work must maintain attribution to the author(s) and the published article's title, journal citation, and DOI. states in general, with single electrons, molecules, and nanoparticles, differing in mass by more than 9 orders of magnitude.

The inelastic Kapitza-Dirac (KD) effect was first discussed in [19]. It differs from its elastic counterpart [20-29] not only by the use of laser fields with different frequencies, but also by the presence of a harmonic trap. The latter modifies the conditions for energy and momentum conservation. The inelastic KD effect is similar to stimulated Raman scattering [30-33] but operates without internal states, so that absorption, spontaneous emission, and decoherence in objects with broad resonance lines can be avoided. It can thus be applied to particles that do not exhibit any internal states at all.

In this Letter, we introduce the Kapitza-Dirac blockade as a new feature emerging from the quantum mechanical treatment of the inelastic KD effect. By using judiciously chosen laser frequencies, the Kapitza-Dirac blockade can drive a controlled parametric resonance while blocking undesired transitions almost entirely.

We start for simplicity with a particle traveling through a 1D harmonic trap (see Fig. 1). The scheme preferably starts from the harmonic oscillator ground state, which can be populated with high probability by filling the trap with a tightly collimated particle beam.

The two KD-laser pulses with frequencies $\omega_{1,2}$ $\left(\omega_{1}>\omega_{2}\right)$ and a $1 / e$ pulse duration $\tau_{\mathrm{KD}}$ are assumed to propagate counter to each other along the $x$ axis. Their polarization should be chosen to avoid wave mixing with the trapping lasers. 


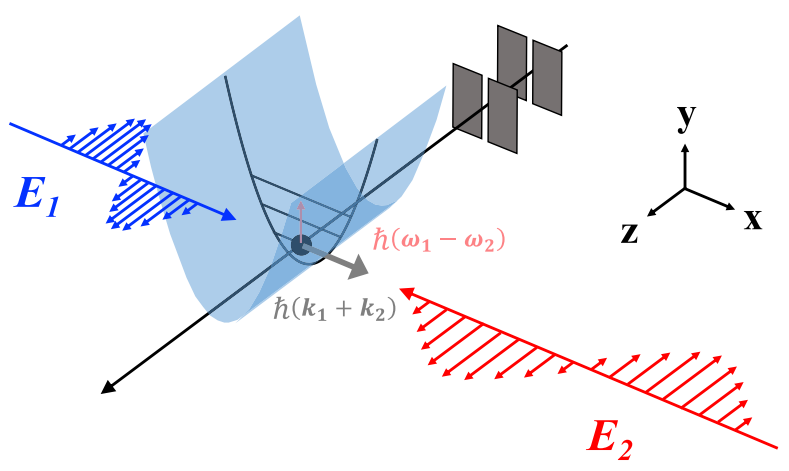

FIG. 1. Proposed realization of the Kapitza-Dirac blockade and quantum state control in a 1D harmonic oscillator. A collimated particle populates the ground state of a 1D harmonic trap. A pair of counterpropagating bichromatic KD-laser fields $E_{1,2}$ interacts with the particle and changes its energy and momentum while it is in the trap.

The oscillator can be parametrically driven through an effective Hamiltonian [34]

$$
\hat{H}_{\text {int }}=\mathcal{C} E_{1}(t) E_{2}(t) \cos \left[\left(k_{1}+k_{2}\right) x-\left(\omega_{1}-\omega_{2}\right) t\right],
$$

where $\mathcal{C}$ is the system specific coupling coefficient, $k_{1,2}=\omega_{1,2} / c$, and $E_{1,2}(t)=E_{1,2} \exp \left(-t^{2} / \tau_{\mathrm{KD}}^{2}\right)$ is the time envelope of the KD-laser pulse. Resonance occurs when

$$
\left\{\begin{array}{l}
\omega_{1}-\omega_{2}=N_{m} \Omega_{0} \\
k_{1}+k_{2}=\left(N_{m}+\delta_{p}\right) k_{0}
\end{array}\right.
$$

where $\Omega_{0}$ is the harmonic trap frequency, $N_{m}$ is a positive integer, and $\delta_{p}>-N_{m}$. The dimensionless momentum detuning $\delta_{p}$ characterizes the distance of $\hbar\left(k_{1}+k_{2}\right)$ to its maximal value $N_{m} p_{0}=N_{m} \hbar k_{0}$, where $k_{0}=\sqrt{m \Omega_{0} / 2 \hbar}$, before the overlap integral between the momentum wave functions decreases (see Fig. 2).

The resonant KD-laser frequencies can be found from Eq. (2) to be $\omega_{1,2}=\omega_{\mathrm{KD}} \pm N_{m} \Omega_{0} / 2$, where the central KD frequency is

$$
\omega_{\mathrm{KD}} \equiv\left(N_{m}+\delta_{p}\right) c k_{0} / 2 .
$$

To demonstrate the preparation of non-Gaussian harmonic oscillator states, we choose $N_{m}=2$ and evaluate the dimensionless transition amplitude $g_{n}(\eta) \equiv\langle n+2| \cos \left[\left(k_{1}+\right.\right.$ $\left.\left.k_{2}\right) x\right]|n\rangle$ for the transition from an oscillator energy eigenstate $|n\rangle$ to $|n+2\rangle[34,38,39]$,

$$
g_{n}(\eta)=-\sqrt{\frac{n !}{(n+2) !}} \eta^{2} L_{n}^{(2)}\left(\eta^{2}\right) e^{-\eta^{2} / 2} .
$$

Here $L_{n}^{(2)}(y)$ is the generalized Laguerre polynomial. The Lamb-Dicke parameter is defined as $\eta \equiv\left(k_{1}+k_{2}\right) x_{0}$, where $x_{0}=\sqrt{\hbar / 2 m \Omega_{0}}$.

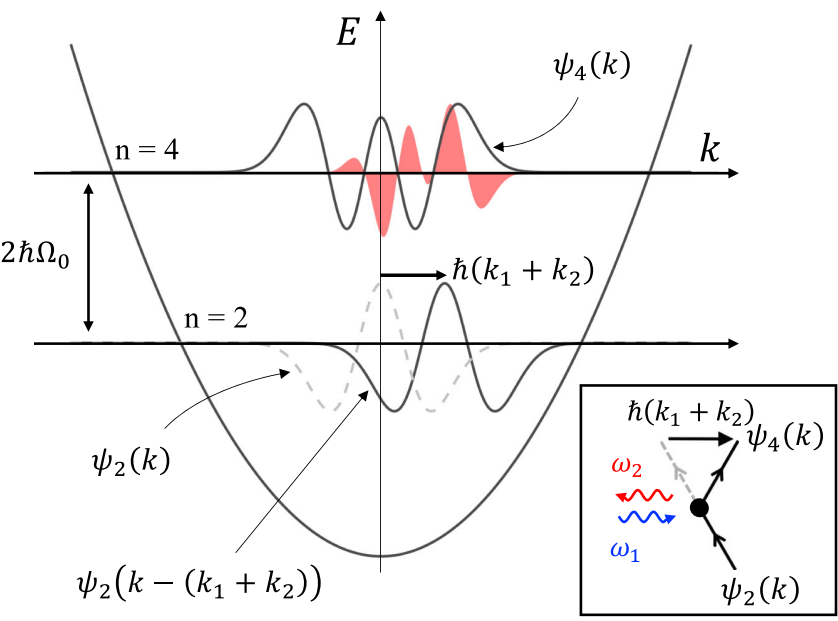

FIG. 2. Kapitza-Dirac blockade in the harmonic oscillator for the transition between the energy eigenstates $|n=2\rangle \rightarrow|n=4\rangle$. Energy and momentum conservation require an energy change of $\hbar\left(\omega_{1}-\omega_{2}\right)=2 \hbar \Omega_{0}$ and a momentum recoil of $\hbar\left(k_{1}+k_{2}\right)=$ $\left(2+\delta_{p}\right) \hbar k_{0}$ (inset). When the overlap integral vanishes, i.e., for $g_{2}(\eta) \propto \int_{-\infty}^{\infty} \psi_{4}^{*}(k) \psi_{2}\left(k-k_{1}-k_{2}\right) d k=0$, this transition is suppressed, even in the presence of resonant laser light. The redshaded integrand $\psi_{4}^{*}(k) \psi_{2}\left(k-k_{1}-k_{2}\right)$ represents the weight of all vertical transition amplitudes starting from different $k$ values. Momentum needs to be tuned for destructive interference to null the overlap integral. The potential is shown with vertical compression for clearer illustration of the wave functions.

The transition amplitude $g_{n}(\eta)$ has zero crossings [see Fig. 3(a)], which implies that the transition $|n\rangle \rightarrow|n+2\rangle$ can be suppressed at certain values of the momentum detuning $\delta_{p}$ because of destructive interference between transition amplitudes starting from different $k$ values (see Fig. 2). This "Kapitza-Dirac blockade" is a powerful tool, as it can stop the sequential excitation in the energy ladder and allows us to prepare non-Gaussian harmonic oscillator states. The energy-momentum conservation Eq. (2) implies that the Lamb-Dicke parameter $\eta=\left(N_{m}+\delta_{p}\right) / 2$ is independent of any oscillator details. In consequence, the Kapitza-Dirac blockade is independent of the specific oscillator realization.

As a first example, we propose to prepare a single energy eigenstate $|n=2\rangle$, starting from the ground state $|n=0\rangle$. Setting $\delta_{p}=0.83$ suppresses the $|n=2\rangle \rightarrow|n=4\rangle$ transition down to $<0.2 \%$ of its maximum value [see Fig. 3(c)]. As a result, transitions starting in $|n=0\rangle$ will end deterministically in $|n=2\rangle$ without populating $|n=4\rangle$ or other excited states in the energy ladder [see Fig. 4(a)]. For a Gaussian-shaped time envelope, complete population inversion occurs when $\Omega_{R} \tau_{\mathrm{KD}}=\sqrt{2 \pi}$, where $\Omega_{R}$ is the system specific Rabi frequency (see Supplemental Material [34]). Note, that $\tau_{\mathrm{KD}}$ should be sufficiently long to keep the pulse bandwidth below the trap frequency $\Omega_{0}$ in order to avoid off-resonant excitation. When the pulse duration is doubled, the Rabi cycle is completed to the ground state 
(a)

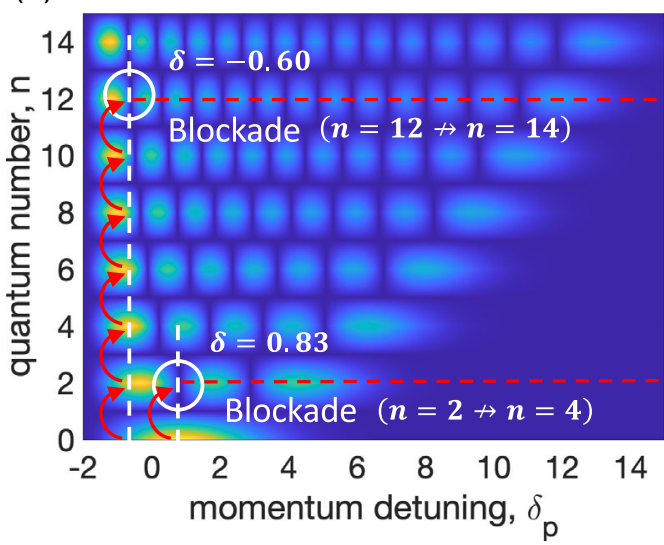

(b)

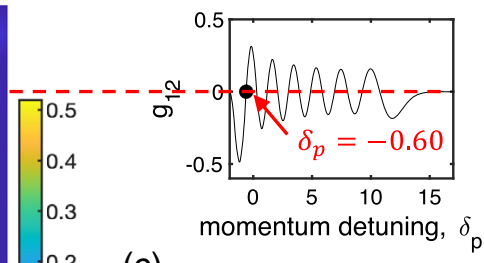

(c)

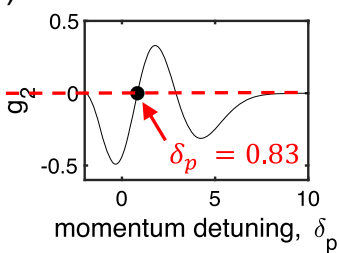

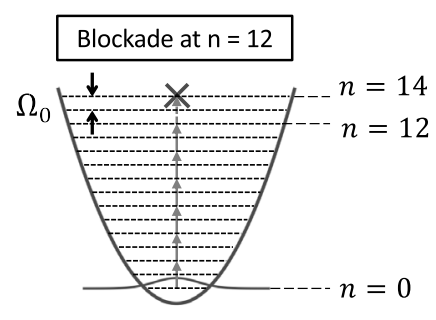

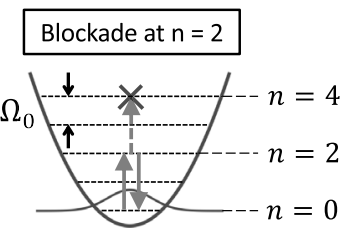

FIG. 3. Transition map for the inelastic KD effect. (a) The norm of the transition amplitude $\left|g_{n}\right|$ in Eq. (4) is plotted as a function of the quantum number $n$ and the momentum detuning $\delta_{p}$. The nodes along the $\delta_{p}$ axis (white circles) can be used to stop sequential excitations (red curved arrows). The width along the $n$ axis does not illustrate the oscillator linewidths but is chosen for clearer illustration of the energy levels. (b) The zero crossing of $g_{12}(\eta)$ at $\delta_{p}=-0.60$ is used to stop transitions beyond $|n=12\rangle$. (c) The zero crossing of $g_{2}(\eta)$ at $\delta_{p}=0.83$ is used to prepare an effective two-level system between $|n=0\rangle$ and $|n=2\rangle$.

[see Fig. 4(b)]. The Kapitza-Dirac blockade has thus transformed the harmonic oscillator into an effective two-level system-with promising applications in quantum information processing.

The Kapitza-Dirac blockade can also prepare a cat state. We show this with a heuristic example of $\delta_{p}=-0.60$,

(a)

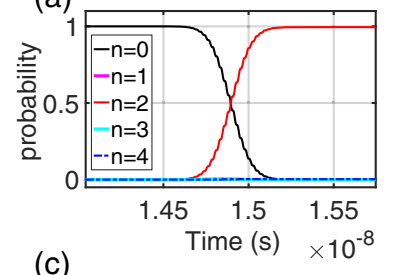

(c)

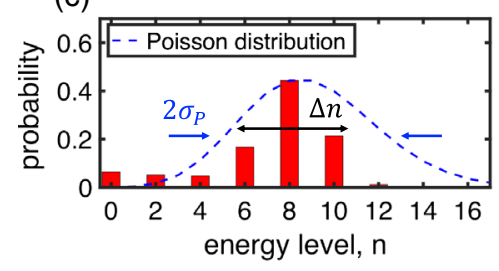

(b)

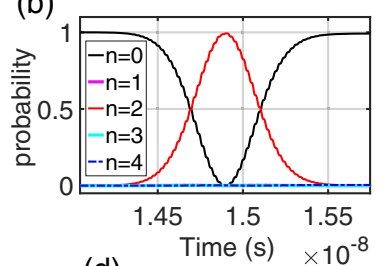

(d)

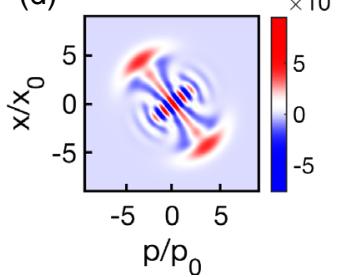

FIG. 4. Kapitza-Dirac blockade as a tool to prepare energy eigenstates and cat states of a harmonic oscillator. (a) Deterministic population transfer to the $|n=2\rangle$ eigenstate can be achieved with a pair of KD-laser pulses. Note that the $|n=4\rangle$ eigenstate (dashed line) is not populated as a result of the Kapitza-Dirac blockade. The small ripples on the probability trace are due to nonresonant excitation at $4 \Omega_{0}$. (b) When the pulse duration in (a) is doubled, the population is coherently returned to the ground state. (c) The population distribution of a cat state has a subPoissonian width $\Delta n<2 \sigma_{P}$, where $\sigma_{P}=\sqrt{n_{\max }}$. (d) The Wigner function of the cat state indicates amplitude squeezing. which suppresses the transition $|n=12\rangle \rightarrow|n=14\rangle$ to $<$ $0.1 \%$ of its maximum value [see Fig. 3(b)]. The oscillator then undergoes sequential excitation from $|n=0\rangle$ up to $|n=12\rangle$ in steps of $\Delta n=2$, but $|n=14\rangle$ is not excited. The pulse intensity $I_{\mathrm{KD}}$ and duration $\tau_{\mathrm{KD}}$ are adjusted together to maximize the population distribution at $n_{\max }=8$ while avoiding off-resonant excitation. The width of the final population distribution is sub-Poissonian [see Fig. 4(c)], due to the Kapitza-Dirac blockade. This leads to an amplitude-squeezed cat state, which is identified by inspecting the Wigner function in Fig. 4(d). The maximum spatial and momentum separation of the cat state, $\Delta x_{\text {cat }}$ and $\Delta p_{\text {cat }}$, are

$$
\frac{\Delta x_{\text {cat }}}{x_{0}}=\frac{\Delta p_{\text {cat }}}{\hbar k_{0}} \approx 4 \sqrt{n_{\text {max }}} .
$$

Substituting Eq. (2) to Eq. (5) with $N_{m}=2$, the number of photon recoils is

$$
N_{\mathrm{ph}}=\frac{\Delta p_{\mathrm{cat}}}{\hbar\left(k_{1}+k_{2}\right)} \approx \frac{4 \sqrt{n_{\mathrm{max}}}}{2+\delta_{p}},
$$

which is again independent of the oscillator properties. If we take $n_{\max }=650$ and $\delta_{p}=-1.8$, the maximum momentum separation is $\Delta p_{\text {cat }} \approx 1000 \hbar k_{532}$, where $k_{532} \equiv 2 \pi / 532 \mathrm{~nm}$.

The Kapitza-Dirac blockade is therefore a promising tool for realizing an all-optical large-momentum-transfer (LMT) beam splitter $[9,10,40,41]$. Moreover, the amplitude-squeezed cat state leaves the divided beam rather well collimated. A Kapitza-Dirac-LMT beam splitter used in conjunction with an optical Bragg grating $[22,27,42]$ could 

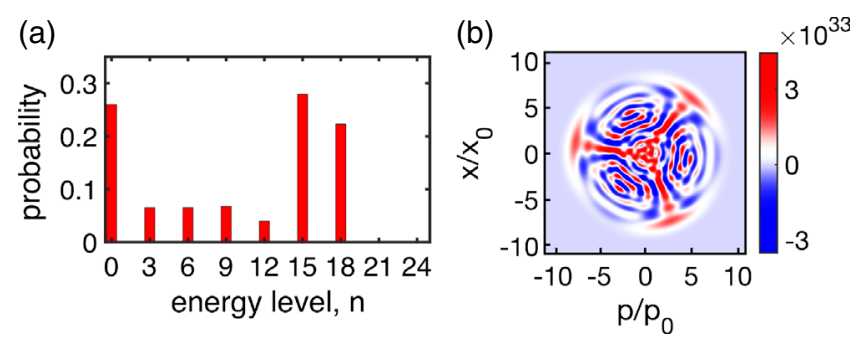

FIG. 5. A three-component cat state. (a) A population distribution for a three-component cat state can be prepared by taking $N_{m}=3$ and $\delta_{p}=-1.57$ in Eq. (2). (b) The Wigner function of the three-component cat state shows interference fringes between each pair and among all three components.

facilitate large-area matter-wave interferometry, in the future.

Also, multicomponent cat states [43-45] can be prepared by properly choosing $N_{m}$ and $\delta_{p}$ [34]. An example is given in Fig. 5 where a three-component cat state is prepared. The KD-laser frequencies $\omega_{1,2}$ in this case are determined by taking $N_{m}=3$ and $\delta_{p}=-1.57$, which suppresses the transition $|n=18\rangle \rightarrow|n=21\rangle$.

Similarly, Gaussian states such as a vacuum-squeezed state $\left(N_{m}=2\right)$ or coherent state $\left(N_{m}=1\right)$ can be prepared with appropriate $I_{\mathrm{KD}}$ and $\delta_{p}$.

The Kapitza-Dirac blockade holds universally. Here we start the experimental discussion with the example of an electron beam 1D trapped by the ponderomotive potential of a standing light wave [46]. Close to the potential minimum, the potential can be approximated as a harmonic trap $U_{p}(x) \approx\left(q_{e}^{2} I_{S} / 2 \epsilon_{0} c^{3} m_{e}\right) x^{2}$, where $m_{e}$ and $q_{e}$ are the electron mass and charge, and $I_{S}$ is the standing wave intensity. The ponderomotive trap frequency is

$$
\Omega_{0}=\sqrt{\frac{q_{e}^{2} I_{S}}{\epsilon_{0} c^{3} m_{e}^{2}}} .
$$

The intensity of each trapping laser that makes the standing wave is $I_{\mathrm{TL}}=I_{S} / 4$. The trap ground state can be populated by a well-collimated electron beam with transverse kinetic energy $m v_{x}^{2} / 2 \ll \hbar \Omega_{0} / 2$.

If we consider the LMT beam splitter example above, using parameters as in Table I, and if we tune the dynamics such that the electron reaches maximum momentum separation when it leaves the trap (see Fig. 1), it will leave in two distinct wave packets separated by $171 \mu \mathrm{m}$ in real space, already $1 \mathrm{~mm}$ behind the trap [47-49].

All aspects discussed above can equally be realized with neutral massive particles with the experimental schemes similar to that of Fig. 1: in Table I we discuss as the second example a porphyrin derivative (TPPF84) with high mass and high vapor pressure [50]. The third example is a silicon
TABLE I. Proposed parameters for preparing large-amplitudesqueezed cat states. The trapping laser for electrons has a $1 / e$ pulse duration of $\tau_{\mathrm{TL}}=0.75 \mathrm{~ns}$ and a repetition rate of $10 \mathrm{~Hz}$. The trapping lasers for molecules and nanoparticles are continuous waves. The momentum detunings for electron, molecule (TPPF84), and nanoparticle $\left(\mathrm{SiO}_{2}\right)$ are $\delta_{p}=-1.8,-1.93$, and -1.93 , respectively. $\langle P\rangle$ is the average laser power.

\begin{tabular}{lccc}
\hline \hline Parameters & Electron & TPPF84 & $\mathrm{SiO}_{2}$ nanoparticle \\
\hline$m(\mathrm{u})$ & $5.49 \times 10^{-4}$ & $2.81 \times 10^{3}$ & $10^{6}$ \\
$\alpha\left(\mathrm{Cm}^{2} / \mathrm{V}\right)$ & $\ldots$ & $2.22 \times 10^{-38}$ & $8.18 \times 10^{-36}$ \\
$\Omega_{0}(\mathrm{rad} / \mathrm{s})$ & $3.22 \times 10^{12}$ & $3.13 \times 10^{4}$ & $1.75 \times 10^{3}$ \\
$\lambda_{\mathrm{TL}}(\mu \mathrm{m})$ & 1.064 & 10.5 & 5 \\
$W_{y-\mathrm{TL}}(\mu \mathrm{m})$ & 100 & 20 & 20 \\
$W_{z-\mathrm{TL}}(\mathrm{mm})$ & 1.6 & 5 & 9 \\
$\left\langle P_{\mathrm{TL}}\right\rangle(\mathrm{W})$ & 37.6 & 30 & 0.037 \\
$I_{S}\left(\mathrm{~W} / \mathrm{m}^{2}\right)$ & $8 \times 10^{16}$ & $15 \times 10^{8}$ & $10.4 \times 10^{5}$ \\
$v_{z}(\mathrm{~m} / \mathrm{s})$ & $6 \times 10^{6}$ & 0.1 & 0.02 \\
$\lambda_{\mathrm{KD}}(\mathrm{nm})$ & 533 & 6819 & 1530 \\
$W_{y-\mathrm{KD}}(\mu \mathrm{m})$ & 100 & 10 & 100 \\
$W_{z-\mathrm{KD}}(\mathrm{mm})$ & 1.2 & 3 & 8 \\
$\left\langle P_{\mathrm{KD}}\right\rangle(\mathrm{mW})$ & 400 & 280 & 1.5 \\
$I_{\mathrm{KD}}\left(\mathrm{W} / \mathrm{m}^{2}\right)$ & $2.6 \times 10^{15}$ & $1.2 \times 10^{7}$ & $2.3 \times 10^{3}$ \\
$\tau_{\mathrm{KD}}(\mathrm{s})$ & $8 \times 10^{-11}$ & $1 \times 10^{-2}$ & $1.4 \times 10^{-1}$ \\
$n_{\mathrm{sca}}$ & $3.9 \times 10^{-3}$ & $8.8 \times 10^{-5}$ & $7.3 \times 10^{-1}$ \\
\hline \hline
\end{tabular}

dioxide $\left(\mathrm{SiO}_{2}\right)$ nanoparticle with low absorption of infrared trapping light. For molecules and nanoparticles, a harmonic trap can be realized by the dipole potential of a standing wave. With $\lambda_{\mathrm{TL}}$ as the trapping laser wavelength, the dipole trap frequency is

$$
\Omega_{0}=\sqrt{\frac{4 \pi^{2} \alpha I_{S}}{\epsilon_{0} c m \lambda_{\mathrm{TL}}^{2}}},
$$

where $\alpha$ and $m$ are the particle's polarizability and mass.

There are three criteria for choosing experimental parameters. First, the laser conditions should satisfy $\tau_{\mathrm{TL}} \gg W_{z-\mathrm{TL}} / 2 v_{z} \gg W_{z-\mathrm{KD}} / 2 v_{z} \gg \tau_{\mathrm{KD}} \gg 2 \pi / \Omega_{0} \quad$ [34], where $\tau$ is the $1 / e$ pulse duration, $W_{z}$ is the $1 / e$ beam diameter along the $z$ axis, and $v_{z}$ is the particle speed in the $z$ direction. Second, the number of Rayleigh scattered photons should be small, $n_{\text {sca }}<1$, to avoid decoherence and dephasing. Third, the central KD frequency $\omega_{\mathrm{KD}}$ should be in the visible or the infrared regime because ultraviolet light would be absorbed in most materials. This implies that lower trap frequencies are preferred for more massive particles. The proposed experimental parameters for large-amplitude-squeezed cat states are listed in Table I, designed according to the empirical formulas

$$
n_{\mathrm{bk}} \approx \frac{2}{3}\left(\frac{2 \pi}{2+\delta_{p}}\right)^{2}
$$



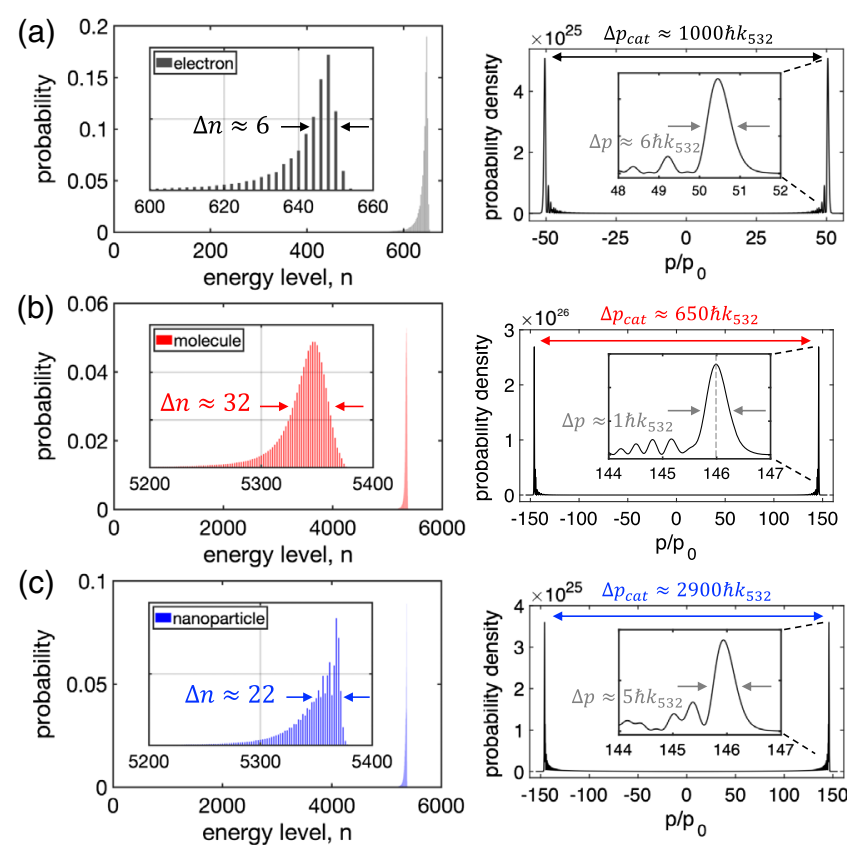

FIG. 6. Simulated maximum momentum separation of amplitude-squeezed cat states, for parameters as in Table I. (a) Electron, $\Delta p_{\text {cat }} \approx 1000 \hbar k_{532}$. (b) Molecule, $\Delta p_{\text {cat }} \approx 650 \hbar k_{532}$. (c) Nanoparticle, $\Delta p_{\text {cat }} \approx 2900 \hbar k_{532}$. The population distributions for electron, molecule, and nanoparticle peak at $n_{\max }=648$, 5348, and 5368, respectively.

$$
I_{\mathrm{KD}} \tau_{\mathrm{KD}} \approx \frac{\mathcal{D}}{\mu_{0}} \sqrt{\frac{32 n_{\mathrm{bk}} \hbar m \Omega_{0}}{\pi}}
$$

where $\left|n_{\mathrm{bk}}\right\rangle$ is the blockade state and the transition $\left|n_{\mathrm{bk}}\right\rangle \rightarrow$ $\left|n_{\mathrm{bk}}+2\right\rangle$ is suppressed. The system specific coefficient $\mathcal{D}$ is $m_{e} \omega_{\mathrm{KD}} / q_{e}^{2}$ for electrons or $1 / \alpha \omega_{\mathrm{KD}}$ for molecules and nanoparticles. The momentum transfer $\Delta p_{\text {cat }}$ is well comparable with the state of the art in matter-wave beam splitting $[9,10,27,40,41]$, but goes beyond it, as the concept can be applied universally to any $1 \mathrm{D}$ trapped particle that scatters light coherently. Additionally, the use of the Kapitza-Dirac blockade yields narrow momentum distributions and avoids overlap between the supposedly distinct wave packets.

The simulations were performed on a supercomputer using time-dependent Schrödinger equations [34,51]. The results are shown in Fig. 6. The momentum transfer $\Delta p_{\text {cat }}$ is $1000 \hbar k_{532}$ for electrons, $650 \hbar k_{532}$ for molecules, and $2900 \hbar k_{532}$ for nanoparticles. The highest eigenstate available for excitation is determined by the onset of trap anharmonicity (see Supplemental Material [34]). In the nanoparticle simulation, the maximal spatial separation in the trap is $\Delta x_{\text {cat }} \approx 1.2 \mu \mathrm{m}$. The required beam velocities for molecules and nanoparticles are 2 orders of magnitude lower than the state of the art of free beams. However, cooling of molecules and nanoparticles is a rapidly advancing field and the proposed parameters are within reach [52-56].

In conclusion, we propose to use the Kapitza-Dirac blockade for manipulating the motional quantum states of single electrons, large molecules, and dielectric nanoparticles. The state preparation scheme is universal, applicable for different particles, and independent of trap details and dimensions.

Our simulations demonstrate the experimental feasibility of preparing various non-Gaussian states and largeamplitude-squeezed cat states. The latter has applications for all-optical LMT beam splitters in matter-wave interferometry. All these results explicitly rely on the coherent but destructive interference in the harmonic oscillator transition amplitudes in the presence of bichromatic light fields: the Kapitza-Dirac blockade.

The proposed control scheme can also be employed for trapped ions and neutral atoms without invoking their internal states. In 2D or 3D harmonic traps one also finds entanglement among different motional degrees of freedom [34]. This can complement existing methods in quantum computing and quantum simulation.

The authors thank Aephraim M. Steinberg, Peter W. Milonni, Christopher Monroe, and Uroš Delić for advice and discussions. W. C. H. wishes to give a special thanks to Yanshuo Li for support and helpful discussions. This work utilized high-performance computing resources from the Holland Computing Center of the University of Nebraska. Funding for this work comes from NSF PHY-1912504.

*waynehuang1984@gmail.com

Present address: University of Göttingen, IV. Physical Institute, 370077 Göttingen, Germany

[1] L. S. Brown and G. Gabrielse, Geonium theory: Physics of a single electron or ion in a Penning trap, Rev. Mod. Phys. 58, 233 (1986).

[2] D. Hanneke, S. Fogwell, and G. Gabrielse, New Measurement of the Electron Magnetic Moment and the Fine Structure Constant, Phys. Rev. Lett. 100, 120801 (2008).

[3] D. Leibfried, R. Blatt, C. Monroe, and D. Wineland, Quantum dynamics of single trapped ions, Rev. Mod. Phys. 75, 281 (2003).

[4] E. A. Cornell and C. E. Wieman, Nobel Lecture: BoseEinstein condensation in a dilute gas, the first 70 years and some recent experiments, Rev. Mod. Phys. 74, 875 (2002).

[5] U. Delić, M. Reisenbauer, K. Dare, D. Grass, V. Vuletić, N. Kiesel, and M. Aspelmeyer, Cooling of a levitated nanoparticle to the motional quantum ground state, Science 367, 892 (2020).

[6] F. Tebbenjohanns, M. L. Mattana, M. Rossi, M. Frimmer, and L. Novotny, Quantum control of a nanoparticle optically levitated in cryogenic free space, arXiv:2103.03853.

[7] M. Arndt and K. Hornberger, Testing the limits of quantum mechanical superpositions, Nat. Phys. 10, 271 (2014).

[8] M. Zawisky, M. Baron, R. Loidl, and H. Rauch, Testing the world's largest monolithic perfect crystal neutron 
interferometer, Nucl. Instrum. Methods Phys. Res., Sect. A 481, 406 (2002).

[9] T. Kovachy, P. Asenbaum, C. Overstreet, C. A. Donnelly, S. M. Dickerson, A. Sugarbaker, J. M. Hogan, and M. A. Kasevich, Quantum superposition at the half-metre scale, Nature (London) 528, 530 (2015).

[10] M. Gebbe, S. Abend, J.-N. Siemß, M. Gersemann, H. Ahlers, H. Müntinga, S. Herrmann, N. Gaaloul, C. Schubert, K. Hammerer, C. Lämmerzahl, W. Ertmer, and E. M. Rasel, Twin-lattice atom interferometry, Nat. Commun. 12, 2544 (2021).

[11] Y. Y. Fein, P. Geyer, P. Zwick, F. Kialka, S. Pedalino, M. Mayor, S. Gerlich, and M. Arndt, Quantum superposition of molecules beyond $25 \mathrm{kDa}$, Nat. Phys. 15, 1242 (2019).

[12] J. Bateman, S. Nimmrichter, K. Hornberger, and $H$. Ulbricht, Near-field interferometry of a free-falling nanoparticle from a point-like source, Nat. Commun. 5, 4788 (2014).

[13] A. Bassi, K. Lochan, S. Satin, T. P. Singh, and H. Ulbricht, Models of wave-function collapse, underlying theories, and experimental tests, Rev. Mod. Phys. 85, 471 (2013).

[14] E. Joos and H. D. Zeh, The emergence of classical properties through interaction with the environment, Z. Phys. B. 59, 223 (1985).

[15] W. K. Wootters and W. H. Zurek, Complementarity in the double-slit experiment: Quantum nonseparability and a quantitative statement of Bohr's principle, Phys. Rev. D 19, 473 (1979).

[16] W. H. Zurek, Decoherence and the transition from quantum to classical, Phys. Today 44, No. 10, 36 (1991).

[17] S. Bose, A. Mazumdar, G. W. Morley, H. Ulbricht, M. Toroš, M. Paternostro, A. A. Geraci, P. F. Barker, M. S. Kim, and G. Milburn, Spin Entanglement Witness for Quantum Gravity, Phys. Rev. Lett. 119, 240401 (2017).

[18] C. Marletto and V. Vedral, Gravitationally Induced Entanglement between Two Massive Particles is Sufficient Evidence of Quantum Effects in Gravity, Phys. Rev. Lett. 119, 240402 (2017).

[19] W. C. Huang and H. Batelaan, Testing quantum coherence in stochastic electrodynamics with squeezed Schrödinger cat states, Atoms 7, 42 (2019).

[20] P. L. Kapitza and P. A. M. Dirac, The reflection of electrons from standing light waves, Math. Proc. Cambridge Philos. Soc. 29, 297 (1933).

[21] D. L. Freimund, K. Aflatooni, and H. Batelaan, Observation of the Kapitza-Dirac effect, Nature (London) 413, 142 (2001).

[22] D. L. Freimund and H. Batelaan, Bragg Scattering of Free Electrons Using the Kapitza-Dirac Effect, Phys. Rev. Lett. 89, 283602 (2002).

[23] P. L. Gould, G. A. Ruff, and D. E. Pritchard, Diffraction of Atoms by Light: The Near-Resonant Kapitza-Dirac Effect, Phys. Rev. Lett. 56, 827 (1986).

[24] P. J. Martin, B. G. Oldaker, A. H. Miklich, and D. E. Pritchard, Bragg Scattering of Atoms from a Standing Light Wave, Phys. Rev. Lett. 60, 515 (1988).

[25] T. Pfau, S. Spälter, Ch. Kurtsiefer, C. R. Ekstrom, and J. Mlynek, Loss of Spatial Coherence by a Single Spontaneous Emission, Phys. Rev. Lett. 73, 1223 (1994).
[26] O. Nairz, B. Brezger, M. Arndt, and A. Zeilinger, Diffraction of Complex Molecules by Structures Made of Light, Phys. Rev. Lett. 87, 160401 (2001).

[27] C. Brand, F. Kiałka, S. Troyer, C. Knobloch, K. Simonović, B. A. Stickler, K. Hornberger, and M. Arndt, Bragg Diffraction of Large Organic Molecules, Phys. Rev. Lett. 125, 033604 (2020).

[28] N. Talebi and C. Lienau, Interference between quantum paths in coherent Kapitza-Dirac effect, New J. Phys. 21, 093016 (2019).

[29] O. Schwartz, J. J. Axelrod, S. L. Campbell, C. Turnbaugh, R. M. Glaeser, and H. Müller, Laser phase plate for transmission electron microscopy, Nat. Methods 16, 1016 (2019).

[30] M. Kasevich and S. Chu, Atomic Interferometry using Stimulated Raman Transitions, Phys. Rev. Lett. 67, 181 (1991).

[31] M. Kozuma, L. Deng, E. W. Hagley, J. Wen, R. Lutwak, K. Helmerson, S. L. Rolston, and W. D. Phillips, Coherent Splitting of Bose-Einstein Condensed Atoms with Optically Induced Bragg Diffraction, Phys. Rev. Lett. 82, 871 (1999).

[32] A. Hemmerich, C. Zimmermann, and T. W. Hänsch, Multiphoton Transitions in a Spin-Polarized 3D Optical Lattice, Phys. Rev. Lett. 72, 625 (1994).

[33] C. Monroe, D. M. Meekhof, B. E. King, S. R. Jefferts, W. M. Itano, D. J. Wineland, and P. Gould, ResolvedSideband Raman Cooling of a Bound Atom to the 3D Zero-Point Energy, Phys. Rev. Lett. 75, 4011 (1995).

[34] See Supplemental Material at http://link.aps.org/ supplemental/10.1103/PhysRevLett.126.253601 for derivations and further discussions, which includes Refs. [35-37].

[35] C. C. Gerry and P. L. Knight, Introductory Quantum Optics (Cambridge University Press, New York, 2004).

[36] H. Batelaan, The Kapitza-Dirac effect, Contemp. Phys. 41, 369 (2000).

[37] W. H. Press, S. A. Teukolsky, W. T. Vetterling, and B. P. Flannery, Numerical Recipes in Fortran 77: The Art of Scientific Computing, 2nd ed. (Cambridge University Press, Cambridge, 1993).

[38] K. E. Cahill and R. J. Glauber, Ordered expansions in boson amplitude operators, Phys. Rev. 177, 1857 (1969).

[39] D. J. Wineland, C. Monroe, W. M. Itano, B. E. King, D. Leibfried, D. M. Meekhof, C. Myatt, and C. Wood, Experimental primer on the trapped ion quantum computer, Fortschr. Phys. 46, 363 (1998).

[40] S.-w. Chiow, T. Kovachy, H.-C. Chien, and M. A. Kasevich, $102 \hbar k$ Large Area Atom Interferometers, Phys. Rev. Lett. 107, 130403 (2011).

[41] J. Rudolph, T. Wilkason, M. Nantel, H. Swan, C. M. Holland, Y. Jiang, B. E. Garber, S. P. Carman, and Jason M. Hogan, Large Momentum Transfer Clock Atom Interferometry on the $689 \mathrm{~nm}$ Intercombination Line of Strontium, Phys. Rev. Lett. 124, 083604 (2020).

[42] D. M. Giltner, R. W. McGowan, and S. A. Lee, Atom Interferometer Based on Bragg Scattering from Standing Light Waves, Phys. Rev. Lett. 75, 2638 (1995).

[43] B. Vlastakis, G. Kirchmair, Z. Leghtas, S. E. Nigg, L. Frunzio, S. M. Girvin, M. Mirrahimi, M. H. Devoret, and R. J. Schoelkopf, Deterministically encoding quantum 
information using 100-photon Schrödinger cat states, Science 342, 607 (2013).

[44] M. Hofheinz, H. Wang, M. Ansmann, R. C. Bialczak, E. Lucero, M. Neeley, A. D. O’Connell, D. Sank, J. Wenner, John M. Martinis, and A. N. Cleland, Synthesizing arbitrary quantum states in a superconducting resonator, Nature (London) 459, 546 (2009).

[45] K. G. Johnson, J. D. Wong-Campos, B. Neyenhuis, J. Mizrahi, and C. Monroe, Ultrafast creation of large Schrödinger cat states of an atom, Nat. Commun. 8, 697 (2017).

[46] H. Batelaan, Colloquium: Illuminating the Kapitza-Dirac effect with electron matter optics, Rev. Mod. Phys. 79, 929 (2007).

[47] A. Caprez, R. Bach, S. McGregor, and H. Batelaan, A wideangle electron grating bi-prism beam splitter, J. Phys. B 42, 165503 (2009).

[48] F. Hasselbach, Progress in electron- and ion-interferometry, Rep. Prog. Phys. 73, 016101 (2010).

[49] F. S. Yasin, K. Harada, D. Shindo, H. Shinada, B. J. McMorran, and T. Tanigaki, A tunable path-separated electron interferometer with an amplitude-dividing grating beamsplitter, Appl. Phys. Lett. 113, 233102 (2018).

[50] S. Gerlich, S. Eibenberger, M. Tomandl, S. Nimmrichter, K. Hornberger, P. J. Fagan, J. Tüxen, M. Mayor, and M. Arndt,
Quantum interference of large organic molecules, Nat. Commun. 2, 263 (2011).

[51] W. C. Huang and H. Batelaan, Discrete excitation spectrum of a classical harmonic oscillator in zero-point radiation, Found. Phys. 45, 333 (2015).

[52] S. Deachapunya, P. J. Fagan, A. G. Major, E. Reiger, H. Ritsch, A. Stefanov, H. Ulbricht, and M. Arndt, Slow beams of massive molecules, Eur. Phys. J. D 46, 307 (2008).

[53] D. Patterson and J. M. Doyle, A slow, continuous beam of cold benzonitrile, Phys. Chem. Chem. Phys. 17, 5372 (2015).

[54] M. R. Tarbutt, Laser cooling of molecules, Contemp. Phys. 59, 356 (2018).

[55] P. Asenbaum, S. Kuhn, S. Nimmrichter, U. Sezer, and M. Arndt, Cavity cooling of free silicon nanoparticles in high vacuum, Nat. Commun. 4, 2743 (2013).

[56] S. Kuhn, G. Wachter, F. Wieser, J. Millen, M. Schneider, J. Schalko, U. Schmid, M. Trupke, and M. Arndt, Nanoparticle detection in an open-access silicon microcavity, Appl. Phys. Lett. 111, 253107 (2017).

Correction: The terminology for cat states has been made consistent throughout the Letter. 